OPEN ACCESS

Edited by:

Mathew Samuel Crowther,

University of Sydney, Australia

Reviewed by:

Federico Morelli,

Czech University of Life Sciences

Prague, Czechia

Catherine Price,

University of Sydney, Australia

${ }^{*}$ Correspondence: Craig Stephen

cstephen@cwhc-rcsf.ca

Specialty section:

This article was submitted to

Urban Ecology,

a section of the journal

Frontiers in Ecology and Evolution

Received: 03 May 2018 Accepted: 03 August 2018 Published: 30 August 2018

Citation:

Stephen C (2018) Evolving Urban

Wildlife Health Surveillance to

Intelligence for Pest Mitigation and

Monitoring. Front. Ecol. Evol. 6:127.

doi: 10.3389/fevo.2018.00127

\section{Evolving Urban Wildlife Health Surveillance to Intelligence for Pest Mitigation and Monitoring}

\author{
Craig Stephen* \\ Canadian Wildlife Health Cooperative, Western College of Veterinary Medicine, University of Saskatchewan, Saskatoon, SK, \\ Canada
}

This paper introduces the concept of harm reduction-based health intelligence as the next step in the evolution of urban wildlife surveillance. There are three reasons to evolve urban wildlife health surveillance: (1) proactive steps to reduce vulnerability to health and safety impacts requires an understanding of environments and social structures as well as of the abundance and distribution of animals or hazards; (2) a hazard-by-hazard approach to surveillance causes management to be reactive rather than proactive; and (3) growing interest in urban wildlife ecology, conservation, and welfare plus the growing recognition of the value of urban wildlife for human well-being requires surveillance to be interested in protecting wildlife health as well as human health. Three strategies to help evolve urban wildlife surveillance to health intelligence are; (1) expand from only tracking a single species or a single threat to also tracking factors that increase the vulnerability of the pests and people in a shared urban setting; (2) be integrative and recognize that multiple concurrent harmful things are affecting people, pests and other species in their shared environments; and (3) develop new collaborative approaches to prevent or mitigate persistent harms from persistent pests without eliminating the pests. This article proposes that harm reduction-based intelligence will better equip city planners and pest managers to identify opportunities to act in advance of significant and concurrent harms to people, infrastructure, and wildlife.

Keywords: urban, wildlife, health intelligence, surveillance, harm reduction

Our attitudes toward wild animals determine if we consider them a pest or as species to be tolerated and even conserved (Delibes-Mateos et al., 2011; Jones et al., 2012; Russell, 2014). For example, protecting rat welfare is of paramount importance in scientific research (Kaliste, 2004) yet our attitudes to urban rats are usually much less magnanimous. Rats that are free-ranging in undisturbed habitats, such as the rock-rat (Zyzomys palatalis) in Australia, are subjects of conservation efforts (Brook et al., 2002) whereas urban brown rats (Rattus norvegicus) are frequently the target of eradication efforts (Capizzi et al., 2014). In the world of urban wildlife health, a quick Google search will show most attention focuses on the role of rats as sources of hazards to other species, such as secondary poisoning of raptors or rodent-borne zoonotic infections. The literature rarely focuses on health for the inherent sake of the rat. Kirkwood and Sainsbury (1996) identified four factors that influence attitudes toward wildlife; (i) the extent to which we are responsible for harm to them; (ii) the extent to which the harmed animals are under our stewardship; (iii) the severity of the problem the wildlife face and (iv) cultural and economic factors, including the popularity of the species involved. The authors noted the illogical but heavily 
weighted role popularity plays. It is, therefore, easy to understand why wildlife health surveillance rarely prioritizes efforts to protect and promote the health of urban wildlife, which we so often consider to be pests rather than wildlife.

Wildlife health surveillance has historically focused on generating early warning signals of risks to society or on tracking specific infectious diseases of concern for wildlife managers (Stephen and Duncan, 2017). There is a long history of wildlife serving as bio-sentinels for the effects and distribution of environmental pollutants and pathogens (Kuiken et al., 2005; Reif, 2011). Wildlife disease surveillance has been used as a guide to predict and prevent new zoonotic disease risks to the public (Stitt et al., 2007). Despite these successes, there is a growing dissatisfaction with the use of wildlife surveillance, largely in reaction to adverse events. Instead, there are new expectations to produce signals to protect wildlife and human health concurrently and proactively.

There are three reasons to reconsider how we design and use urban wildlife health surveillance. First, changes in urban wildlife social structures and habitats are known to determine wildlife vulnerability to environmental hazards (ex. Bradley and Altizer, 2007; Lee et al., 2018). Tracking clues of changing vulnerability may allow more targeted and proactive actions to avoid impacts on public health and safety as well as to protect wildlife health. Managers may be better able to take precautionary steps when vulnerability is tracked, as opposed to focussing only on the abundance and distribution of animals or hazards. Second, a hazard-by-hazard approach to risk management is insufficient to reduce vulnerability and promote resilience to emerging risks. It dooms management strategies to remain reactive rather than proactive. In an era of unprecedented social, landscape, and climate changes, emerging risks are the norm, reducing the usefulness of surveillance that only tracks known hazards (Stephen et al., 2015). Third, growing interest in urban wildlife ecology, conservation, and welfare (ex. Adams, 2005) and growing recognition of the value of urban wildlife areas and biodiversity for human well-being (Soulsbury and White, 2016) suggest that better information is needed to reciprocally promote the health of wildlife and people in urban environments. Protection of the health of one species should not come at an unsustainable or unacceptable expense to another. Shifting the target of attention away from threats and hazards to health and vulnerability may allow for earlier interventions and open more options for surveillance and action.

Wildlife health is increasingly being viewed as the cumulative effect of social, physical, and biological influences on the capacity of individuals and populations to; (i) access their needs for daily living; (ii) have capacity to cope with and adapt to stressors and change and (iii) meet ecological and social expectations (Nordenfelt, 2011; Hanisch et al., 2012; Stephen, 2014). Wildlife health programs rarely assembled all three aspects into a complete picture. As such, control programs tend to be fragmented and inefficient, or worse, unintentionally impede each other. For example, it has been proposed that rodent control programs focussed on lethal population reduction may increase the prevalence of zoonotic pathogens in some urban rat populations (Lee et al., 2018). Wildlife health surveillance usually does not track factors influencing population vulnerability, leaving that instead to population ecologists who in turn do not take full advantage of health surveillance information.

A significant impediment to wildlife surveillance is the lack of agreement on the indicator threshold that signals the need for an intervention. It is generally accepted that human endeavors should not unnecessarily compromise wild animal welfare (Kirkwood and Sainsbury, 1996), but the threshold for "unnecessary compromise" is ill-defined. It is also unclear if that threshold is different for urban and non-urban wildlife. For example, some stakeholders might have zero risk tolerance and demand eradication of infections from an urban wild population while others see an animal's microbiological flora as part of our biodiversity heritage and thus their associated risks better controlled by managing the human dimensions of risk. First principles of population health (ex. Karpati et al., 2002; Gowan et al., 2014) argue for surveillance that focuses on the determinants of health and vulnerability rather than only on the presence and distribution of hazards, but knowledge gaps preclude evidence-based recommendations on the best variables to monitor in urban wildlife. Moreover, divergent social values complicate finding consensus on thresholds that meet varying risk perceptions.

There are three strategies to evolving wildlife health surveillance to meet the changing landscape of urban wildlife risk to both address growing concerns about the equitable protection of human and urban wildlife health, and to provide more integrated signals to support a precautionary, rather than reactionary, urban pest management approach. First, surveillance needs to evolve from tracking a single threat to an intelligence system that not only tracks hazards and adverse outcomes but also tracks factors that increase the vulnerability of the pests and people in a shared urban setting. For example, the reasons for failure or success of wildlife health management are usually social, rather than biological or physical factors. Therefore, an urban wildlife health intelligence system needs to be able to characterize changes in human dimensions of risk (including changes to the built environment), social conditions affecting human exposure or susceptibility to wildlife-associated harms, and changes in risk perception. At the same time attention must be paid to variables that create cumulative stressors on urban wildlife that increase their vulnerability to new hazards. Research will be needed to specify and prioritize determinants of vulnerability for a given location. However, a general wildlife health intelligence system needs to be able to gather information on; (i) the biological endowment of the population (ex diseases and stressors); (ii) the animal's social environment (ex. extent of competition and demographics); (iii) the quality and abundance of the needs for daily living (ex. food supplies and habitat availability); (iv) their abiotic environment (ex. climate variables such as floods); (vi) sources of direct mortality (ex. lethal pest control), and (vii) changing human expectations (ex. social attitude and municipal policies). This strategy is compatible with ecological-based pest management (Singleton et al., 1999) as well as bio-economic approaches to rodent pest control (Stenseth et al., 2003). It is also compatible with opinions that ecological-based pest management must attend to social and 
cultural dimensions to ensure pest control efforts are adopted and sustained (Palis et al., 2007).

Second, an urban wildlife health intelligence system needs to recognize that multiple concurrent harms are affecting people, pests, and other species in their shared environments. Over abundant rodents, for example, can cause various social harms (ex fear, zoonotic disease transmissions and damage to housing infrastructure); harm rodent predators and scavengers (ex. impacts of rodenticides on the safety and availability of prey) and impact the rodents themselves (ex. reduced welfare through intra-specific competition and increased non-zoonotic rodent diseases). The importance of each harm will vary between stakeholders, but it is inevitable that their interconnections and interdependencies will create unique challenges and opportunities for intervention. Recognizing the ties between society, nature, and technology has been proposed as a better way to create a comprehensive, cohesive pest management strategy that is ecologically sound and socially acceptable (Mougenot and Roussel, 2005).

Third, given that cities can find it very difficult to eliminate their urban wildlife problems on a sustainable basis (Fernández et al., 2007; Himsworth et al., 2013), new collaborative approaches are needed to find interventions that can prevent or mitigate persistent harms from persistent pests without eliminating the pest entirely.

The various harms created by urban wildlife are embedded in complex socio-ecological systems. Therefore, multiple points of view are needed to characterize and respond to urban pest problems. Because underlying, incessant social conditions can cause pest problems to persist, there is often a feeling of helplessness in efforts to eliminate their negative impacts. As we move from individual animals to the population and ecosystem level, risk management decision-making becomes more variable, uncertain, and complex. The inseparable links between the individual, social and ecologic levels of harm from urban wildlife suggest that multi-level interventions are required to make increment gains in the health of wildlife, human communities and their shared ecosystems. Harm reduction is a set of perspectives and processes that might offer insights into how to evolve urban wildlife health surveillance to this end.

Harm reduction is most often used to describe a set of public health strategies to reduce the harmful consequences of addictive behaviors on individuals and society (Hunt et al., 2003). It acknowledges that society is unlikely to eliminate substances like illicit drugs and that attempts at elimination have been insufficient to prevent the harms arising from addiction. Like illicit drugs, urban pests and their associated hazards are often persistent problems that are hard to eradicate. Harm reduction programs aim to prevent or reduce adverse consequences to all community members rather than only targeting the hazardous substance or circumstance. Harm reduction applies to all the determinants of health and not merely problematic risks. It involves pragmatic approaches to remove barriers to implementing knowledge to protect health and promote sustainability. Harm reduction aims to decrease the impact on multiple actors in a community (in this case human and animals) by addressing both the amount of harm and its impacts (Marlatt,
1996). The amount of harm can be reduced by reducing exposure to and sensitivity of a population to hazards whereas the impacts are addressed by promoting populations' capacity to cope with the harm and by addressing harms as cumulative effects (Stephen et al., 2018b).

An urban wildlife health intelligence program based on harm reduction would need to adopt the following six principles (based on $\mathrm{BCMOH}^{1}$ ). First, collect data on the nature and distribution of the social, health, and animal harms as opposed to only tracking the causes of harms such as pathogens or diseases. Second, recognize that pest or their diseases are not likely to be eliminated in the short term and therefore seek information on variables that can pragmatically be manipulated to reduce animal and human vulnerability to pest related harms. Third, develop an information network that will reveal feasible options to help populations cope with existing harms within the current circumstances while efforts to eliminate these harms are ongoing. Fourth, health intelligence signals need to identify and prioritize actions that can produce incremental gains that can be built on over time. Fifth, recognize that actions attacking one harm may have unintended consequences for other harms, necessitating integrated analysis. Finally, health intelligence systems need to be people oriented and actively engage a diversity of players to find pathways and control points that can be targeted to reduce harms across various perspectives, priorities, and values.

Figure 1 illustrates the relationship between health intelligence and harm reduction. Health intelligence helps to set priorities for harm reduction actions. Health intelligence uses surveillance to create situational awareness of harmful substances or circumstances along with reconnaissance to characterize the local conditions that may impede or enable interventions. The total amount of harm caused by urban wildlife is affected by the total harms across social and animal domains and the impacts of those harms. A socio-ecological assessment of harms helps identify targets for health intelligence.

Harm reduction place decisions makers' needs at the forefront of their design and implementation (Regmi et al., 2016). As there are multiple layers of decision makers in pest management, from the household to senior government officials, a harm reductionbased health intelligence system needs a good human network to ensure analysts understand the information needs, priorities, and thresholds across the decision-making spectrum. Contextual understanding helps turn information into intelligence and increases the likelihood that surveillance and reconnaissance results will be put into action (Haines et al., 2004). As much attention needs to be placed on human intelligence and information networks as on datasets to turn diverse sets of information into coherent intelligence outputs.

Harm reduction-based health intelligence should help people make informed decisions and empower them to minimize harms by identifying ways to reduce negative impacts until a hazard can be moderated or eliminated. Harm reduction

\footnotetext{
${ }^{1}$ British Columbia Ministry of Health (BCMOH). Harm Reduction: a British Columbia Community Guide. British Columbia Ministry of Health. Available online at: http://www.health.gov.bc.ca/library/publications/year/2005/ hrcommunityguide.pdf (Accessed May 5, 2018).
} 


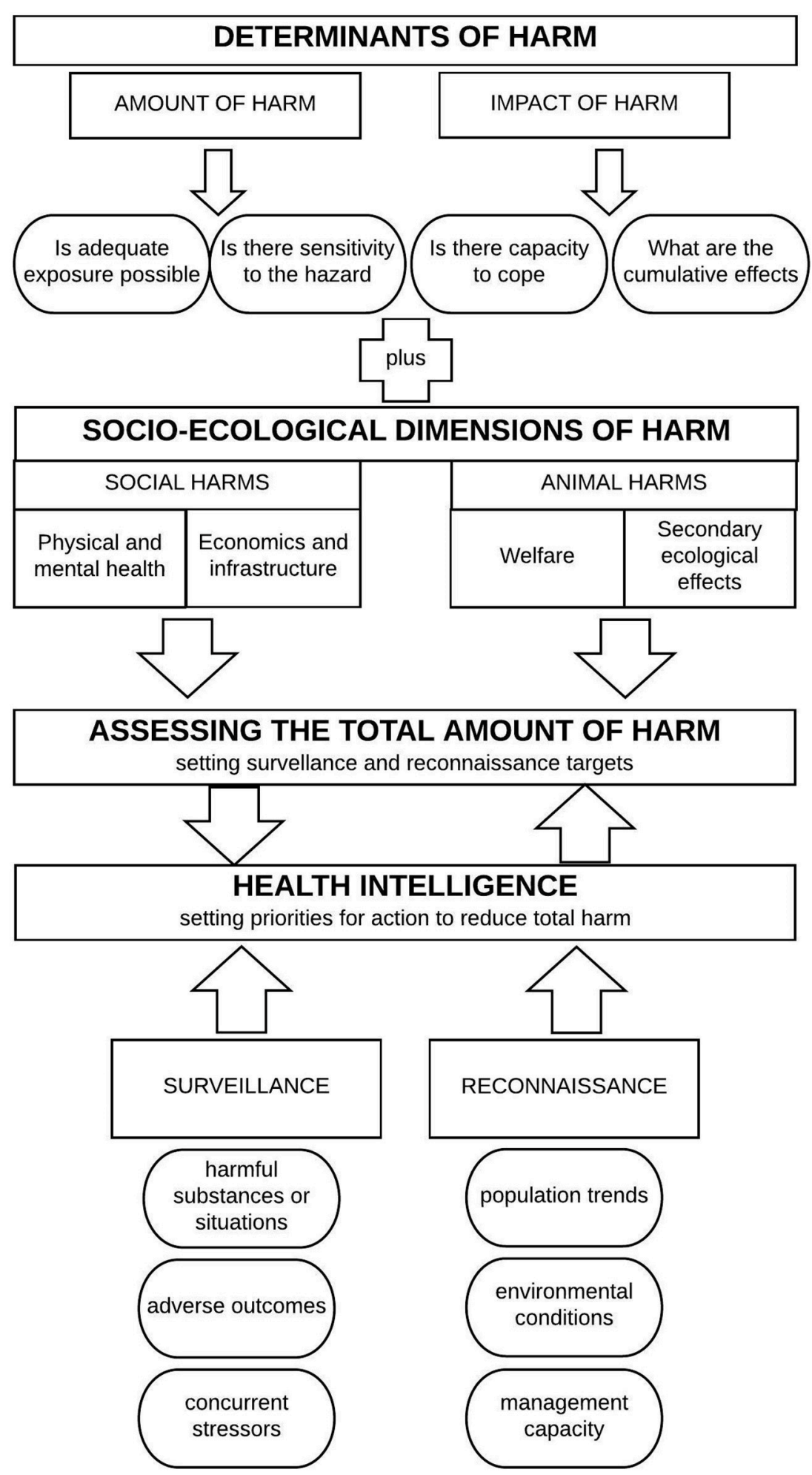

FIGURE 1 | Conceptual model of the integration of health intelligence and harm reduction to set priorities for urban wildlife health surveillance and action.

promotes collaborative policy and action by discovering means for horizontal, cooperative approaches to protecting health in advance of serious, irreversible impacts. Adopting a harm reduction perspective is not a rejection of the current surveillance paradigm but rather a call to expand our scope of observations to ensure that opportunities to lessen harms can be identified 
and acted on while we strive to directly prevent or avoid the negative consequences of urban wildlife. Decision-makers need to understand the full scope of a pest problem and how to; (i) recognize priority problems and needs, (ii) track progress to evaluate the impact of interventions, and (iii) make evidence-based decisions on policy, program design and resource allocation (Regmi et al., 2016).

It is important to note the word "evolving" in the title of this paper. The scope of most wildlife health programs is more limited than what I am proposing above. Neither literature nor legislation describe the necessary features of an urban wildlife health intelligence system. Stephen et al. (2018a) have proposed a generic set of attributes of national wildlife health surveillance programs that share features with those described in this paper particularly; (i) the expectation to integrate health into risk management planning and assessment, (ii) an interest in the determinants of health as well as health outcomes, and (iii) the need to engage players outside of traditional wildlife health sectors. But their recommendations emphasized free-ranging non-urban wildlife which are more often of concern to trade and conservation. An urban wildlife health intelligence approach needs local information and context to identify feasible and acceptable strategies to reduce pest-associated harms. It is often beyond the capacity of departments responsible for local pest control to have the expertise and human resources to gather all the necessary information by themselves. Strategic partners to enable collective intelligence across multiple departments such as public health, urban planning, and sanitation would be needed. Investment should focus on centralized capacity to collect, integrate, and assess data already being gathered by other departments, supplemented with scientific literature and insights gathered through participatory processes.

Working examples of an urban health intelligence system do not yet exist in the literature but examples exist in other sectors. Epidemic intelligence and public health observatories are public health approaches that use an intelligence approach (Hemmings and Wilkinson, 2003; Paquet et al., 2006). Both track multiple lines of information on hazards, hosts and environments to identify vulnerabilities and threats before they impact populations. The Canadian Wildlife Health Cooperative has adopted these concepts to evolve its surveillance database to an intelligence platform. Its ongoing work is exploring how

\section{REFERENCES}

Adams, L. W. (2005). Urban wildlife ecology and conservation: a brief history of the discipline. Urban Ecosyst. 8, 139-156. doi: 10.1007/s11252-0054377-7

Biermann, F. (2007). 'Earth system governance' as a crosscutting theme of global change research. Global Environ. Change 17, 326-337. doi: 10.1016/j.gloenvcha.2006.11.010

Bradley, C. A., and Altizer, S. (2007). Urbanization and the ecology of wildlife diseases. Trends Ecol. Evol. 22, 95-102. doi: 10.1016/j.tree. 2006.11.001

Brook, B. W., Griffiths, A. D., and Puckey, H. L. (2002). Modelling strategies for the management of the critically endangered Carpentarian rock-rat (Zyzomys palatalis) of northern Australia. J. Environ. Manage. 65, 355-368. doi: 10.1016/S0301-4797(02)90561-X ecological, environmental, and microbiological clues from nature and farms can inform wild bird avian influenza preparedness. This article call for an urban wildlife health intelligence system is a response to the growing need for tools and practices to link diverse data sources to better reflect the origins of and solutions to problems from human-wildlife interactions. However, specifying the precise information to track will depend, in part, on the species being managed, the availability of contextual information and emerging research to identify the most reliable signals. The intent of this paper is to inspire investigation and investment to move this idea from the conception to implementation.

There will always be a need to control urban wildlife. As we become more urbanized, there will also be the need to sustain the benefits of urban wildlife for human well-being. The world is experiencing unprecedented rates of social and environmental changes due to climate change, global movement of people and products, and rapid urbanization (Biermann, 2007; Horton et al., 2014). A new approach is needed to provide the necessary situational awareness to stay on top of the inevitable emerging risks that will arise with these changes. The concept of harm reduction and the needs to expand the realms of wildlife surveillance have been recommended elsewhere (ex. Stephen and Duncan, 2017; Stephen et al., 2018b) and serve as a basis to advocate for the changes discussed in this article. By evolving urban wildlife health surveillance from its tradition of solely looking at the distribution and prevalence of hazards to a harm reduction-based intelligence approach, I propose that city planners and pest managers will be better equipped to identify opportunities and needs to act in advance of significant harms to people, infrastructure and the wildlife that call our cities home.

\section{AUTHOR CONTRIBUTIONS}

The author confirms being the sole contributor of this work and approved it for publication.

\section{FUNDING}

This work was supported by the Canadian Wildlife Health Cooperative. 
Haines, A., Kuruvilla, S., and Borchert, M. (2004). Bridging the implementation gap between knowledge and action for health. Bull. World Health Organ. 82, 724-731.

Hanisch, S., Riley, S., and Nelson, M. (2012). Promoting wildlife health or fighting wildlife disease: insights from history, philosophy and science. Wildl. Soc. Bull. 36, 477-482. doi: 10.1002/wsb.163

Hemmings, J., and Wilkinson, J. (2003). "What is a public health observatory?" J. Epidemiol. Commun. Health 57, 324-326. doi: 10.1136/jech.57.5.324

Himsworth, C. G., Feng, A. Y., Parsons, K., Kerr, T., and Patrick, D. M. (2013). Using experiential knowledge to understand urban rat ecology: a survey of Canadian pest control professionals. Urban Ecosyst. 16, 341-350. doi: 10.1007/s11252-012-0261-4

Horton, R., Beaglehole, R., Bonita, R., Raeburn, J., McKee, M., and Wall, S. (2014). From public to planetary health: a manifesto. Lancet 383:847. doi: 10.1016/S0140-6736(14)60409-8

Hunt, N., Ashton, M., Lenton, S., Mitcheson, L., Nelles, B., and Stimson, G. (2003). A Review of the Evidence-Base for Harm Reduction Approaches to Drug Use. London: Forward Thinking on Drugs.

Jones, C., Barron, M., Warburton, B., Coleman, M., Lyver, P. O. B., Nugent, G. (2012). Serving two masters: reconciling economic and biodiversity outcomes of brushtail possum (Trichosurus vulpecula) fur harvest in an indigenous New Zealand forest. Biol. Conserv. 153, 143-152. doi: 10.1016/j.biocon.2012. 04.016

Kaliste, E. (2004). The Welfare of Laboratory Animals, Vol. 2. Dordrecht: Springer Science and Business Media.

Karpati, A., Galea, S., Awerbuch, T., and Levins, R. (2002). Variability and vulnerability at the ecological level: implications for understanding the social determinants of health. Am. J. Public Health 92, 1768-1772. doi: 10.2105/AJPH.92.11.1768

Kirkwood, J. K., and Sainsbury, A. W. (1996). Ethics of interventions for the welfare of free-living wild animals. Anim. Welfare 5, 235-244.

Kuiken, T., Leighton, F. A., Fouchier, R. A., LeDuc, J. W., Peiris, J. S., and Schudel, A., et al. (2005). Public health. Pathogen surveillance in animals. Science 309, 1680-1681 doi: 10.1126/science. 1113310

Lee, M. J., Byers, K. A., Donovan, C. M., Bidulka, J. J., Stephen, C., Patrick, D. M., et al. (2018). Effects of culling on Leptospira interrogans carriage by rats. Emerg. Infect. Dis. 24, 356-360. doi: 10.3201/eid2402.171371

Marlatt, G. A. (1996). Harm reduction: come as you are. Addict. Behav. 21, 779-788. doi: 10.1016/0306-4603(96)00042-1

Mougenot, C., and Roussel, L. (2005). To poison or to trap? The ecologisation of 'pest'control. Soc. Ruralis 45, 115-129. doi: 10.1111/j.1467-9523.2005. 00294.x

Nordenfelt, L. (2011). Health and welfare in animals and humans. Acta Biotheora. 59, 139-152. doi: 10.1007/s10441-011-9125-1

Palis, F. G., Singleton, G., Sumalde, Z., and Hossain, M. (2007). Social and cultural dimensions of rodent pest management. Integr. Zool. 2, 174-183. doi: 10.1111/j.1749-4877.2007.00057.x

Paquet, C., Coulombier, D., Kaiser, R., and Ciotti, M. (2006). Epidemic intelligence: a new framework for strengthening disease surveillance in Europe. Euro Surveill. 11, 212-214. doi: 10.2807/esm.11.12.00665-en
Regmi, K., Bendel, N., and Gee, I. (2016). "Public health intelligence: an overview," in Public Health Intelligence, eds K. Regmi and I. Gee (New York, NY: Springer International Publishing), 1-18.

Reif, J. S. (2011). Animal sentinels for environmental and public health. Public Health Rep. 126 (Suppl 1), 50-57. doi: 10.1177/0033354911 $1260 S 108$

Russell, J. C. (2014). A comparison of attitudes towards introduced wildlife in New Zealand in 1994 and 2012. J. R. Soc. N. Z. 44, 136-151. doi: 10.1080/03036758.2014.944192

Singleton, G. R., Leirs, H., Hinds, L. A., and Zhang, Z. (1999). Ecologically-Based Management of Rodent Pests-Re-Evaluating Our Approach to an Old Problem. Canberra: Australian Centre for International Agricultural Research (ACIAR), 17-29.

Soulsbury, C. D., and White, P. C. (2016). Human-wildlife interactions in urban areas: a review of conflicts, benefits and opportunities. Wildl. Res. 42, 541-553. doi: 10.1071/WR14229

Stenseth, N. C., Leirs, H., Skonhoft, A., Davis, S. A., Pech, R. P., Andreassen, H. P., et al. (2003). Mice, rats, and people: the bio-economics of agricultural rodent pests. Front. Ecol. Environ. 1, 367-375. doi: 10.1890/15409295(2003)001\%5b0367:MRAPTB\%5d2.0.CO;2

Stephen, C. (2014). Toward a modernized definition of wildlife health. J. Wildl. Dis 50, 427-430. doi: 10.7589/2013-11-305

Stephen, C., Berezowski, J., and Misra, V. (2015). Surprise is a neglected aspect of emerging infectious disease. Ecohealth 12, 208-211. doi: $10.1007 /$ s10393-014-1001-4

Stephen, C., and Duncan, C. (2017). Can wildlife surveillance contribute to public health preparedness for climate change? A Canadian perspective. Clim. Change 141, 259-271. doi: 10.1007/s10584-016-1892-x

Stephen, C., Sleeman, J., Nguyen, N., Zimmer, P., Duff, J. P., Gavier-Widén, D., et al. (2018a). Proposed attributes of national wildlife health programmes. Rev. Sci. Tech. 37.

Stephen, C., Wittrock, J., and Wade, J. (2018b). Using a harm reduction approach in an environmental case study of fish and wildlife health. Ecohealth 15, 4-7. doi: 10.1007/s10393-017-1311-4

Stitt, T., Mountifield, J., and Stephen, C. (2007). Opportunities and obstacles to collecting wildlife disease data for public health purposes: results of a pilot study on Vancouver Island, British Columbia. Can. Vet. J. 48, 83-90.

Conflict of Interest Statement: The author declares that the research was conducted in the absence of any commercial or financial relationships that could be construed as a potential conflict of interest.

The reviewer CP and handling Editor declared their shared affiliation

Copyright (c) 2018 Stephen. This is an open-access article distributed under the terms of the Creative Commons Attribution License (CC BY). The use, distribution or reproduction in other forums is permitted, provided the original author(s) and the copyright owner(s) are credited and that the original publication in this journal is cited, in accordance with accepted academic practice. No use, distribution or reproduction is permitted which does not comply with these terms. 